\title{
Towards radical innovations in a mature company: an empirical study on the UX-FFE model
}

\section{Adrien Lecossier ${ }^{1}$, Marc Pallot ${ }^{2}$, Pascal Crubleau ${ }^{3}$ and Simon Richir ${ }^{2}$}

\section{Research Paper}

LAMPA, 2 Boulevard du Ronceray, 49100 Angers, FRANCE

Cite this article: Lecossier A, Pallot $M$ Crubleau P, Richir S (2019). Towards radical innovations in a mature company: an empirical study on the UX-FFE model. Artificial Intelligence for Engineering Design, Analysis and Manufacturing 1-16. https://doi.org/ $10.1017 / \mathrm{S} 0890060419000118$

Received: 29 August 2018

Accepted: 30 October 2018

\section{Key words:}

Assessment; co-creation; front end innovation; fuzzy front end; ideation; innovation management; mixed-methods; reliability; user eXperience

Author for correspondence:

Adrien Lecossier,

E-mail: adrien.lecossier@ensam.eu Innovation, Angers, France

\section{Abstract}

${ }^{1}$ Esterline \& Arts et Métiers ParisTech, LAMPA Presence \& Innovation research team, Angers, France; ${ }^{2}$ Arts et Métiers ParisTech, LAMPA Presence \& Innovation research team, Angers, France and ${ }^{3}$ Université d'Angers, ISTIA

The ability to successfully conduct radical innovations is mandatory for mature industrial companies that want to remain competitive in the global market. This ability relies on several ingredients, namely: (1) the structuring of the innovation process; (2) managerial principles; (3) methodological tools; (4) the presence of a culture of innovation. This paper reports about the impact of applying the User eXperience-Fuzzy Front End (UX-FFE) model, which brings together the systemic innovation process with the social, economical, and methodological aspects on the outcomes of the innovation process. Firstly, it appears that the operational performance of the upstream innovation process relies on the quality of the social context, intrinsic to the group of co-creators, corresponding to the reported perceived experience. Secondly, the UX-FFE model application, therefore, allows optimizing the upstream innovation process performance. Indeed, we argue that the evaluation of the co-creators perceived experience brings new opportunities to optimize the operational performance of the upstream innovation process. The first part of this paper presents deeper a theoretical model, named UX-FFE, which combines a UX approach with an upstream innovation process (FFE). The main interest of this UX-FFE model is that it allows evaluating the social aspect of the upstream innovation process, which may be detrimental to the success of radical innovation projects in mature companies. The second part presents the results of previous experiments that validated the model. The results allow the design of an instrument dedicated to the evaluation of the user experience of co-creators in the ideation stage. Finally, the third part reports about the experimentation of the UX-FFE in a mature company. Results present the impact of the co-creators' experience on the performance of radical innovation projects.

\section{Introduction}

A mature industrial company seeks to maintain a deterministic and profitable model, named dominant design, which was built over time (Utterback, 1994). Therefore, this mature company essentially creates incremental innovations that consist in reducing the costs of existing products while substantially improving their performance in order to preserve their market share (Rolstadås et al., 2012). This is why most of the income of a mature industrial enterprise comes from gains made in the mature ecosystem (E.Porter, 1985). Its economic growth is directly correlated to that of the field of activity in which it operates mainly. A stable and mature company may tend to over-protect its dominant model. This behavior can lose the sense of objectivity when analyzing the changes in its ecosystem. So, in the event of business turbulence or even a questioning of the industrial environment, its comfortable situation can very quickly deteriorate. The most quoted example concerns Kodak, which lost most of its main revenue stream in a few years, refusing to undertake the necessary digital change (Lucas and Goh, 2009). Unfortunately, the same story will happen again and again. These kinds of firm, having a similar mindset, design new products by systematically improving the dominant model as the basis for innovation. This obsession with the improvement and preservation of the existing dominant model through repeating incremental innovations will undoubtedly lead to their failure in missing breakthrough innovation opportunities.

Conducting incremental innovation implies a performance improvement of the dominant design. This kind of improvement can be seen in the technological or marketing aspect of the object that has been optimized. Improving the marketing aspect is achieved by optimizing the performance of the existing business model and the ecosystem. The technological improvement of the object focuses on an increase in performance of its functional description and on the accuracy of its evaluation criteria (Lecossier et al., 2016). In contrast and in order to survive on longer terms, an industrial company has to create radical innovations (Christensen, 1997; Gaia Rubera and Kirca, 2012). A radical innovation allows improving the performance of the dominant design. However, this increase must be 5-10 times greater 
than the existing system or disrupts the dominant design in order to be considered radical (O'Connor and Rice, 2001; Garcia and Calantone, 2002).

The value creation of radical innovation projects emerges in the upstream phase of innovation commonly named Fuzzy Front End (FFE) (Cooper, 2008; Frishammar et al., 2011; Markham, 2013). Many practitioners consider the FFE stage as delicate to manage. In fact, it requires navigating and progressing in the FFE while uncertainties are high and interactions are complex due to the multidisciplinary nature (Kim and Wilemon, 2002). To overcome this challenge, more and more companies are tooling and structuring their upstream process with specific processes and methodologies in order to make it more reliable and efficient (Nicholas, 2014). Interest in the upstream phase of innovation is steadily increasing since it is in this phase that sustainable value creation projects emerge (Frishammar et al., 2011; Markham, 2013).

According to Rajapathirana and Hui, the innovation capability is the most required components for developing effective radical innovation outcomes within a firm. Authors define the innovation capability as an ability to identify and understand future customer needs, expectations, and potential adoption (Rajapathirana and Hui, 2017). Such an innovation capability requires developing an innovation culture for being able to create new ideas and transform them into successful radical innovations (Slater et al., 2014). However, in a mature industrial company the economic, systemic, operational, and social aspects are often blurred; because the solution is not mono-dimensional, a multidisciplinary approach is required to successfully create and validate radical innovation concepts (Baregheh et al., 2009).

Hence, our main objective is to study the upstream stage of the innovation process according to the economic, social, systemic, and operational impacts on the innovation process; in order to allow a mature industrial company, SOURIAU ESTERLINE, to re-engineer its upstream innovation process through a multidisciplinary approach. Indeed, because the economic, systemic, operational, and social aspects are blurred in a mature industrial company, a multidisciplinary approach is required to successfully create and validate breakthrough innovation concepts.

This paper starts with the literature review on Front end of Innovation (FEI), User eXperience (UX), and Co-creation that brings the social side of innovation. Secondly, we introduce our proposed UX-FFE model that brings together the UX approach and the FFE process. Then, we present experiments' findings concerning the validation of our proposed model and its application in an industrial context. Finally, there is a discussion on the limits of our study and conclusion as well as future work.

\section{Existing theories and previous work}

\section{Frond end of innovation}

\section{Systemic approach: the process view}

The idea of demystifying the operational approach of the upper stage of innovation was born at the end of the $90 \mathrm{~s}$.

Today, it is widely admitted that the FFE stage corresponds to a process starting the whole innovation cycle (Cooper, 2001), (Zhang and Doll, 2001). The completed activities, and overtaken decisions during the FFE stage also represent the starting point of the entire innovation cycle. In the case of New Product Development (NPD), the performance of the NPD process directly depends on the work achieved during the FFE stage. This phenomenon is identified as the "leverage effect" (Verworn et al., 2008). Koen and colleagues were the first to structure the FFE stage into a clearer process named "Frond End of Innovation" process (Koen et al., 2001). The FEI process is a set of five steps working iteratively: (1) Opportunity identification; (2) Opportunity analysis; (3) Idea generation; (4) Idea selection; (5) Concept validation.

In view of the interest it represents, the FEI model has since been supplemented and modified several times by other scholars (Reid and De Brentani, 2004), (De Brentani and Reid, 2012). A new step named "Concept Selection" had also been added in order to separate the step "Concept \& Technology Development" in two parts. We retain the version that orders the FEI in three macro-stages and we respectively called: "Strategy", "Ideation", and "Validation" (Lecossier and Pallot, 2017) (Fig. 1). This version is a combination of the original FEI process with the innovation pipeline. The six FFE steps are then positioned inside the macro-stages.

\section{Sociological approach for radical innovation}

According to Koen et al. the cultural innovation engine is the element having the most important impact on the success of the activities realized during the FEI process (Koen et al., 2001), (Koen et al., 2002). This explains that the six FEI steps could be successfully completed if there is a proper cultural innovation engine inside the organization (Rajapathirana and Hui, 2017). Ahmed defines that an organization in which a cultural innovation engine conducive to radical or breakthrough innovation exists, it exhibits properties, such as: employees have initiatives, risk-taking is not sanctioned, and hierarchy is reduced to a minimum (Ahmed, 1998). McLaughlin and al. revealed nine key themes that represent aspects of an innovation culture influencing radical or breakthrough innovation: (1) Freedom/Latitude; (2) Attitude to risk; (3) Growth/Development; (4) Internal Confidence; (5) External Confidence; (6) External Perspective; (7) Clear objective; (8) Team Constitution; (9) Company infrastructure (McLaughlin et al., 2008). More recently, a study shows that there are five main managerial lines to follow at the project level to innovate radically (Pihlajamaa, 2017). The first concerns the project goals that should be moderately specified in order to increase individual motivation as the second which suggests breaking radical innovation projects into the smaller sub. The third managerial line for radical innovation is putting in place resources for projects. Belonging to a project team with diverse means to approach complex problems may increase the individuals' belief in their ability to reach project goals. The fourth guideline consists to compose a project team with a variety of perspectives and expertise in order to increase individual motivation for radical innovation. Finally, the last managerial good practice at the project level is to reduce bureaucracy.

\section{Operational and methodological approaches for radical innovation}

Many methods have been implemented in recent years to equip the FEI process and largely innovation processes (Nicholas, 2014), (Koen et al., 2002). Our literature review carried out on Scopus confirms that there are many methods for each of the FEI steps (Table 1). The following query "(Step of the FEI process AND method AND innovation)" yielded a large number of results for all FEI steps.

\section{Limitations of the previous work}

Many studies are carried out about systemic, sociological, managerial, and operative visions of innovation in order to facilitate 


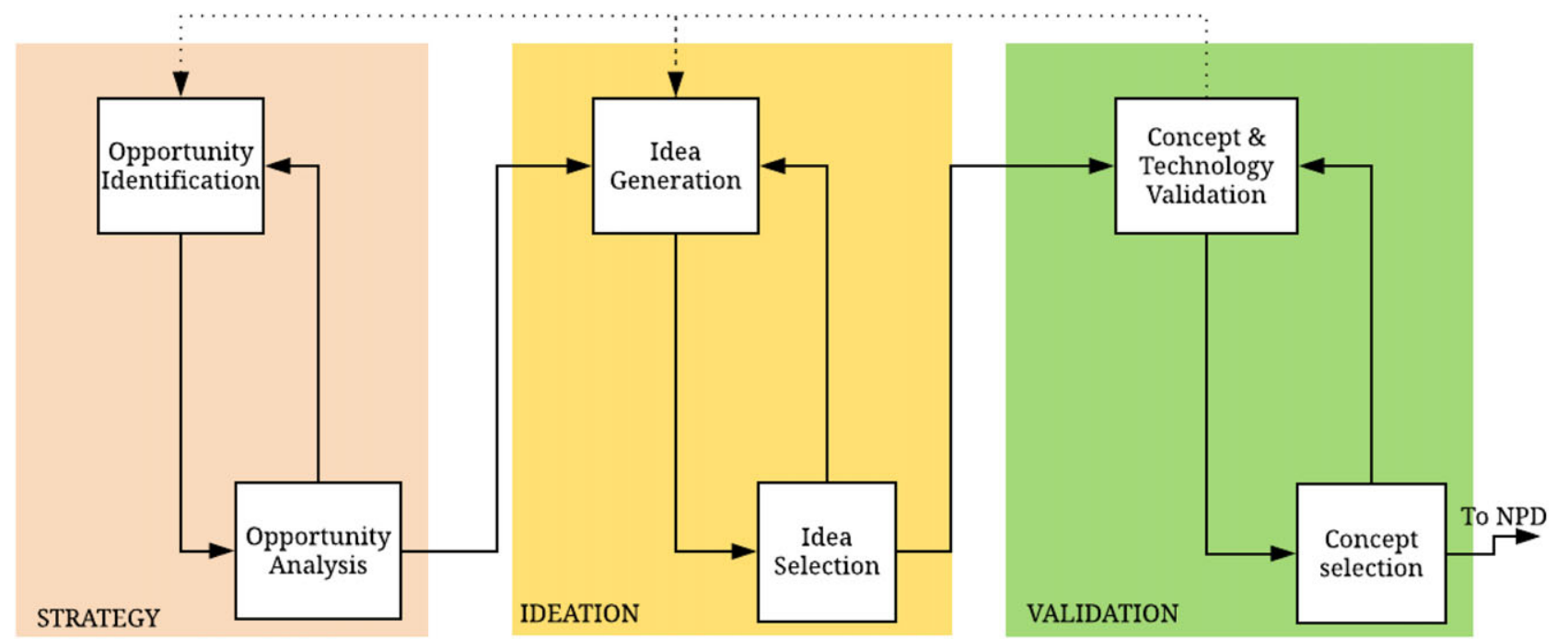

Fig. 1. Reformulated FEI (Lecossier and Pallot, 2017).

Table 1. Number of results to the query "(step of the FEI process and method and innovation)" on Scopus

\begin{tabular}{|c|c|c|c|c|c|}
\hline $\begin{array}{l}\text { Semantic } \\
\text { research }\end{array}$ & $\begin{array}{l}\text { [TITLE-ABS-KEY } \\
\text { (opportunity } \\
\text { identification AND } \\
\text { method)] AND } \\
\text { (Innovation) }\end{array}$ & $\begin{array}{c}\text { [TITLE-ABS-KEY } \\
\text { (opportunity analysis } \\
\text { AND method)] AND } \\
\text { (Innovation) }\end{array}$ & $\begin{array}{l}\text { [TITLE-ABS-KEY(idea } \\
\text { generation AND } \\
\text { method)] AND } \\
\text { (Innovation) }\end{array}$ & $\begin{array}{c}\text { [TITLE-ABS-KEY(idea } \\
\text { selection AND } \\
\text { method)] AND } \\
\text { (Innovation) }\end{array}$ & $\begin{array}{c}\text { [TITLE-ABS-KEY } \\
\text { (concept validation } \\
\text { AND method)] AND } \\
\text { (Innovation) }\end{array}$ \\
\hline $\begin{array}{l}\text { Number of } \\
\text { results }\end{array}$ & 543 & 4104 & 743 & 380 & 540 \\
\hline
\end{tabular}

the creation of radical innovations. However, the different approaches are not put in place with the others. This situation raises several questions:

- Among the sets of methodologies, how many are compatible and respectful of the social factors that are essential to the success of a radical innovation project?

- There is no evidence that the use of these methods in an industrial environment does not decrease the quality of the social factors that are essential to the success of a radical innovation project, such as the absence of bureaucracy. Can we say that the exponential growth in the number of methods and processes dedicated to innovation has no effect on the degradation of the social factors that are essential to the success of a radical innovation project?

- What links do exist between methodological, sociological, and systemic approaches?

- What are the best methodologies to use in each macro-stage of the FEI process in order to increase the economical and social aspects contributing to the innovation performance?

To study these questions, we have proposed a new model called UX-FFE (Lecossier and Pallot, 2017), (Lecossier et al., 2017a), (Lecossier et al., in press). The UX-FFE model is an extension of the FEI process with a UX approach in order to support the sociological viewpoint inside a systemic and operational structuration.

In few words, the UX-FFE model principle consists to ensure that a group of persons, who co-creates a radical innovation, follows the FEI process and applies methodologies could perceive a good quality of experience, ensuring only a positive effect of the process structuration and increasing the performance of breakthrough creation and validation.

\section{User eXperience}

It is today still difficult to find a consensus, between researchers and practitioners, on what UX is (Scapin et al., 2012). For example, Law et al. claim that: "UX is dynamic, context dependent, and subjective." (Law et al., 2009). ISO DIS 9241-210 defines UX as "all aspects of the user's experience when interacting with the product, service, environment or facility" (ISO FDIS 9241-210 2009). The "user is at the center of design" becomes the watchword. Therefore, UX has been in the last years an approach used in numerous disciplines (e.g. Human computer interaction (HCI), ergonomics, information architecture, branding, ...) to improve user-centric products or services. According to Pallot and Pawar, UX is a multi-dimensional and multi-faceted concept reflecting many different types of experience, including social and empathical experiences, that users can live through when using a product/service (Pallot and Pawar, 2012). They also suggested that each type of experience is then decomposed into elements and properties for the evaluation of its perceived quality. For example, the UX-FFE model is composed of six types of experiences (Emotional, Cognitive, Interpersonal, Empathical, Technological, and Economical) corresponding overall to nine elements and 27 properties (Lecossier et al., in press).

\section{Co-creation: the social side of innovation}

Our original approach in this study on radical innovation at the FFE stage in a mature company consists in addressing the social side of innovation rather than focusing only on the economic 
aspects; hence, including the employees' experience of their innovation process in order to assess the social side of innovation. Quite often innovation processes simply focus on economic factors while ignoring social factors (Hart et al., 2015); in fact, the social side of innovation considers the social settings within enterprises' innovation process, either as informal or formal organizations and arrangements (Hart et al., 2012). Pallot et al. have studied co-creation and social immersion as a related form of the social side of the innovation process (Pallot et al., 2012). They argue that innovation paradigms and design approaches promote distributed collaboration among organizations and user communities. However, project stakeholders are mainly trained for improving their individual skills through learning experiences rather than getting a live user experience through socially immersive environments that could unleash their creative potential. Pallot and colleagues discuss issues related to the use of different social interaction strategies for enabling the proper level of co-creation. They also argue that people are trained for enhancing their individual skills and improving their individual productivity rather than for enhancing their social skills and improving their interpersonal productivity. On the one hand, employees, which are users of the innovation process, have to experience something new while sharing feedback, meaning and understanding within the community of involved stakeholders; on the other hand, researchers collect data for better understanding emerging behaviors and usage patterns as well as embedded adoption mechanisms. Consequently, they try to evaluate the role of distributed cognition and collective intelligence, including both social and emotional intelligence in the co-creation activity and decision-making process.

Recently, Rihova et al. introduced a Customer-to-Customer co-creation perspective, conceptualizing the social layers within which value is formed. This approach extends current value co-creation discussions by providing conceptual insights into co-creation within the social sphere of employees and even customers; hence, allows benefiting from understanding how both employees and customers can be "nudged" into more socially immersive co-creation layers (Rihova et al., 2013). The "Living Labs" movement, initiated by the European Commission in 2006 and based on the previous work of W. J. Mitchel from MIT, has identified the power of engaging all stakeholders, especially users, at the earlier stage of innovation for co-creating value elements. According to Pallot, the Living Lab approach is intended to have all project stakeholders (Pallot and Pawar, 2012): (1) co-creating new innovative scenarios in bringing together technology push and market pull (i.e. crowdsourcing, crowdcasting) into a diversity of views, constraints and Knowledge Sharing; (2) exploring emerging usages and behaviors; (3) experimenting and (4) evaluating, including socio-economic aspects, new ideas and innovative concepts as well as related artifacts in real life contexts in order to observe the potential of viral adoption of new artifacts through a confrontation with users' value models.

In contrast with the traditional industrial design of product reliability and affordability, the co-creation strategy does not imply to mainly focus on designing product features but rather on designing the most appropriate experience for ensuring a higher rate of product or service adoption (Dale, 2015). However, anticipating the UX is not something obvious to implement; it requires designers to empathize with users in order to understand the values to be co-created, problems to be solved, and the kind of social interactions that should be facilitated (Pallot and Pawar, 2012). Experience Design aims at designing an overall experience of appropriate usage scenarios. Therefore, exploring patterns of usage and capturing emerging users' behavior become a cornerstone in design iterations that are intended to raise the level of product adoption. It implies to better understand the nature of repetitive or unique experience elements that are, consciously or unconsciously, propagated by all stakeholders driving towards successful innovations. The human and social nature of experience, through its broad diversity, involves several dimensions, namely: Knowledge, Social, Societal, Technological, and Economical, which should be concurrently addressed in a comprehensive and balanced way (Pallot and Pawar, 2012).

During the last decades the role of users in the innovation process has gradually evolved from lead user (Von Hippel, 1986; Von Hippel, 2005) towards user co-creation [(Sanders, 2008); (Sanders and Stappers, 2008); (Mulder and Stappers, 2009); (Pallot and Pawar, 2012); (Ramaswamy and Gouillart, 2010)]. While Sanders and Stappers drafted a domain landscape of design research (Sanders and Stappers, 2008), Mulder and Stappers argued that the notions of co-creation and co-design have been growing within the participatory design landscape (Mulder and Stappers, 2009). They proposed to involve active users by making use of generative techniques in order to practice more the concept of engaging users as co-creation contributors in order to re-enforce the living side of the Living Lab environment. They also stated that co-creation necessitates sharing and collaboration; hence, an open mindset that it not so trivial to put in place. Pallot et al. $(2010 a, 2010 b)$ created a landscape of research and design methods involving users at the earlier stage of the innovation process. This domain landscape was experimented as an evaluation tool for assessing the maturity level, in terms of user involvement in the innovation process, of 14 visited innovation Labs named "Living Labs" due to their specific experimentation nature of reallife context (Salminen et al., 2011).

Ramaswamy and Gouillart consider that Co-creation is about engaging people to create more value together and involves redesigning interactions based on individuals' experiences. They argue that through co-creation, organizations can unleash the creative energy of people, especially employees and internal stakeholders, but also customers, suppliers, and related external stakeholders and communities, to create mutual value (Ramaswamy and Gouillart, 2010). The term "co-creation" is a typical design collaboration activity synonymous to ideation but refers to the engagement of customers/users/citizens for co-creating value (De Brentani and Reid, 2012). Furthermore, co-creation is a group activity, based on social interaction, which implies the notion of co-presence (or social presence) when it occurs in immersive environments. Hence, co-creation is one of the activities characterizing User-Centered Design (UCD) methods, such as eXperience Design $(\mathrm{XD})$. When a group of participants representing different disciplines of stakeholders has to collaborate, distance factors, such as interpersonal distance, affect their co-creation performance (Pallot et al., 2010a, 2010b). One could argue that it is exactly the same for co-creating value elements in socially immersive environments because it is just a particular form of collaboration involving users as experts of usage scenarios. In this context, interpersonal distance is a well-identified social factor impeding co-creation effectiveness and efficiency (Pallot et al., 2012).

\section{UX-FFE model proposal}

The UX-FFE model is an extension of the FEI process with a UX approach in order to support the sociological viewpoint inside a systemic structuration. 


\section{Model principle}

The introducing of UX in our field of research consists to consider the FEI process and its methodologies as systems. This means that we are interested in the feelings of people who use the methodologies and methods of the FEI process. Indeed, while a method is operationally structuring to be effective, its use must in no case give rise to a sense of bureaucracy, under penalty of entering a paradoxical situation.

Finally, the principle of our model proposal consists to ensure that a person who follows the FEI process and apply its methodologies can experience a good quality of experience, avoiding the paradox effect of the process structuration and increasing the performance of breakthrough creation and validation. It allows taking concurrently into account the social and economic aspects necessary for the breakthrough and radical innovation creations.

The UX-FFE model principle supposes to consider that each phase of the process has a capacity $\mathrm{Cx}$ allowing to identify, let emerge and validate radical ideas. This ability can be improved through the use of tools and methods. Each stage of the process produces a result noted Ix corresponding to the radical innovation project progress. Finally, each stage is subject to the social aspect (the cultural innovation engine) that can be considered as a "social voltage" noted UXx.

Therefore, identify and validate UX experience types which allow to evaluate is truly important because of their direct impact on the FEI process performance. It is this research topic that we will treat in this paper. Indeed, despite the amount of research works there is no UX approach dedicated to the interaction of a person with a process and methodology. In fact, most of UX approaches used inside the innovation processes are dedicated to optimize the quality of experience with the product/service that a user is designing. However, we retained the Holistic Model of User Experience (HMUE) as a base because its components are enough general and consequently adaptable to characterize system interactions (Pallot and Pawar, 2012). The HMUE model is composed of four dimensions: Human, Social, Societal, and Business. Each dimension is evaluated by different experience types. For example, the social dimension is evaluated by two experiences: Interpersonal and Emphatical. And these experiences are constituted of element: Social ties, Interaction, and Group dynamics for the Interpersonal experience; Caring for the Emphatical experience. Finally, elements are evaluated by the properties as for example "Sense of community" and "Sense of indulgence" for the Caring element.

Therefore, the HMUE components we want to link with the FEI process should rationally be linked to the cultural engine definition. In order to establish a link, we applied the instantiation method as proposed by Krawczyk et al. (2017).

\section{Instantiated UX model}

The instantiation method consists of checking the correspondence between value elements, to be delivered to the users by the investigated solution (FEI process, methodologies), and UX elements appearing in the holistic UX model. In other words, a subset of relevant UX components and properties from the holistic model is turned into an implemented model according to the characteristics of the proposed solution to be adopted by users. For example, in order to deliver the value of the positive or negative impact caused by a methodology which makes a hierarchical distinction between users it is anticipated that "group dynamics" element is relevant. The reasoning is the same for each of the engine element. One should answer the next questions:

(1) "What is the HMUE dimension which characterizes the studied engine property?"

(2) "In the selected dimension, what is the type of experience corresponding to the engine property description?"

(3) "In the selected type of experience, what is (are) the element (s) of the HMUE allowing to evaluate the engine property?"

(4) "In the selected HMUE element, what is (are) the property (ies) of the HMUE allowing to evaluate the engine property?"

Table 2 presents some matches of the HMUE components with some of the cultural engine elements after the instantiation method has been realized.

The overall UX-FFE construct contains three dimensions, six types of experience, nine elements and 20 properties (Appendix A).

\section{Survey instrument}

In order to ensure that UX components we selected to characterize the UX-FFE model are reliable, we should statically validate them for the three "social voltages" of the UX-FFE model, noted.

From the metrological point of view, the UX measurement is today still immature and not reliable (Lecossier et al., 2017b). Indeed, according to the International Vocabulary of Metrology (i.e. VIM 3), a measurement process should generate "one or more quantity values that can reasonably be attributed to a quantity". Moreover, "the results of the process, by which quantity values have been obtained, are characterized by the metrological compatibility, metrological comparability and, if necessary, metrological traceability".

To face this problematic situation, we decide to use an evaluation method which combines quantitative and qualitative evaluations (Cresswell et al., 2003). According to Krawczyk et al. (2017) the using of a Mixed Method (MM) allows for more confident interpretation of the results as well as early detection of potential problems with the reliability and validity of the UX survey instrument and/or collected data. Therefore, as in their study dedicated to a mobile app, we decided to base our research approach on MM Concurrent Strategies with the qualitative part embedded into the quantitative one.

The instrument is a bipolar survey that embeds an open-ended question for each rating question in order to capture respondents' justifications behind their ratings. Each question that contains the survey should allow evaluating the anticipated properties that constitute the UX-FEE model (Appendix A).

\section{UX-FFE model validation}

The UX-FFE model principle supposes to consider that each phase of the process has a capacity $\mathrm{Cx}$ allowing to identify, let emerge, and validate radical ideas. This ability can be improved through the use of tools and methods. Each stage of the process produces a result noted Ix corresponding to the radical innovation project progress. Finally, each stage is subject to the social aspect (the cultural innovation engine) that can be considered as a "social voltage" noted UXx. 
Table 2. Example of the instantiation method application: links between elements of the cultural engine and the Holistic Model of User Experience

\begin{tabular}{|c|c|c|c|c|}
\hline \multicolumn{2}{|c|}{$\begin{array}{l}\text { Some of the cultural engine components (Ahmed, 1998) (McLaughlin et al., } \\
\text { 2008) }\end{array}$} & \multicolumn{3}{|c|}{$\begin{array}{l}\text { Some of the Holistic Model of User Experience components (Pallot and } \\
\qquad \text { Pawar 2012) }\end{array}$} \\
\hline Engine elements & Element properties & Dimension & Type of experience & Element \\
\hline Organic structures & - Face to face communication; little red tape & \multirow[t]{2}{*}{ Social } & \multirow[t]{2}{*}{ Interpersonal Experience } & \multirow{2}{*}{$\begin{array}{l}\text { - Social ties } \\
\text { - Interaction } \\
\text { - Group dynamics }\end{array}$} \\
\hline Freedom/Attitude to risk & - Allow discussion of dumb ideas & & & \\
\hline
\end{tabular}

\section{Validation of the UXi model}

After using the instantiation method associated with the HMUE, we defined the experiential construction of the UX-FFE model (Lecossier et al., in press). This construction serves as a reference to build the UX evaluation instrument for each stage of the process (UXx for the Strategy stage, UXi for the Ideation stage, and UXv for the Validation stage). Our latest study has allowed to identify a reliable construct of the UXi experience and to build a mixed methods instrument to evaluate it (Lecossier et al., in press) (Fig. 2).

The UXi construct is based on three experience types (Emotional, Interpersonal, and Socio-economical), four elements (Emotional connection, Social Ties, Interaction, and Satisfaction) and 12 properties (Attractiveness, Responsiveness, Fulfilness, Trusted relationship, Openness, Collaboration, Communication, Emerging concepts, Usefulness, Hedonic quality, and Replicability).

Finally, one of our lasted studies shows that the UX of a group of persons who innovate during the ideation stage of the FEI process can be evaluated (Lecossier et al., in press). The primary interest of this ability is to continuously evaluate the quality of the social context in the ideation stage; essential for the success of radical innovation projects.

When evaluation instruments are built for UXs and UXv, it will then be possible to evaluate the social context quality of the group throughout the FEI process

\section{Validation of the UX-FFE principle}

All processes must be controllable and adjustable to be improved. Since it is not possible to optimize without evaluating (Torgerson, 1958), the UX-FFE model incorporates a UX approach to assess the social voltage (social situation) intrinsic to the FEI process performance.

Consequently, the second step in the development of the UX-FFE model was to link the quality of the user experience (UXx) with the operational performance of the project (noted Ix) generated in each phase of the FEI process. As a result, it will become possible to anticipate a performance drift by evaluating the UX perceived by people working on an innovative project during the FEI process. Thus, it becomes possible to implement corrective actions in order to fix any deviations even during the upstream stage of innovation.

In our previous work, we have identified some initial and interesting elements justifying further study of the UX-FFE model. For example, we found that there was a link between the level of satisfaction of the people who participated in one of the stages of the FEI process and their resulting willingness to continue to carry out the innovative project (Lecossier and Pallot, 2017). This emphasized that the hedonic aspect of the innovation process plays an important role in the opportunity to pursue radical innovation projects in a mature firm. We also found that the quality of the collective experience during the validation stage facilitated the maturation of a radical innovation project (Lecossier et al., 2017a).

The experiment presented in our latest study demonstrated that there are links between the UXi perceived by a group that innovates and the operational performance of radical innovation projects (Lecossier et al., 2018a). The UXi perceived by participants in each project is not the same. In the case of radical innovation, the operational performance (Ii) is linked to the quality of the perceived UXi throughout the ideation stage (Fig. 3). Indeed, results showed that if the same method is used for different projects of radical innovation $(\mathrm{Ci}=$ constant $)$, the operational performance variation $(\Delta \mathrm{Ii})$ is linked to the quality of the perceived $\mathrm{UXi}$ during the ideation stage.

We found that the gap between a mostly appropriate UXi (2.69) and a really appropriate UXi (3.06) has a significant impact on the operational performance Ii ("almost performing" in violet vs. "performing" in green in Fig. 3). The limit of this study is that it had not been realized inside an industrial environment but only with groups of students. Therefore, the next step is to apply and validate the UX-FFE model inside a mature industrial company.

\section{Validation of the UX-FFE model}

Since our study is aimed at mature industrial companies, the next step is to verify if the results of the previous studies coincide with those of the experimentation carried out in our pilot company.

The experiment presented in this paper belongs to the last step of development of the UX-FFE model. Its two objectives are to: (1) know the operational elements that impact the UXi of groups of co-creators; (2) observe the links of dependence between the $\mathrm{UXi}$ and the operational performance of an innovation project that is in the ideation stage of the upstream innovation process of our pilot company.

\section{UX-FFE application in the context of a mature company}

\section{Experiment description}

This experiment concerns three radical innovation projects, namely: project A (P1) "printed connector", project B (P2) "attractiveness of the company", and project C (P3) "collaborative workspace". These projects were conducted within the pilot company during our study. For each project the process was the same. The first step was to build a team of volunteers to work on the project. For this, innovation themes were shared with all employees. The most interested could then volunteer to work on one of them.

The launch of the teamwork was done during a first session. The innovation manager used this session to explain how the 


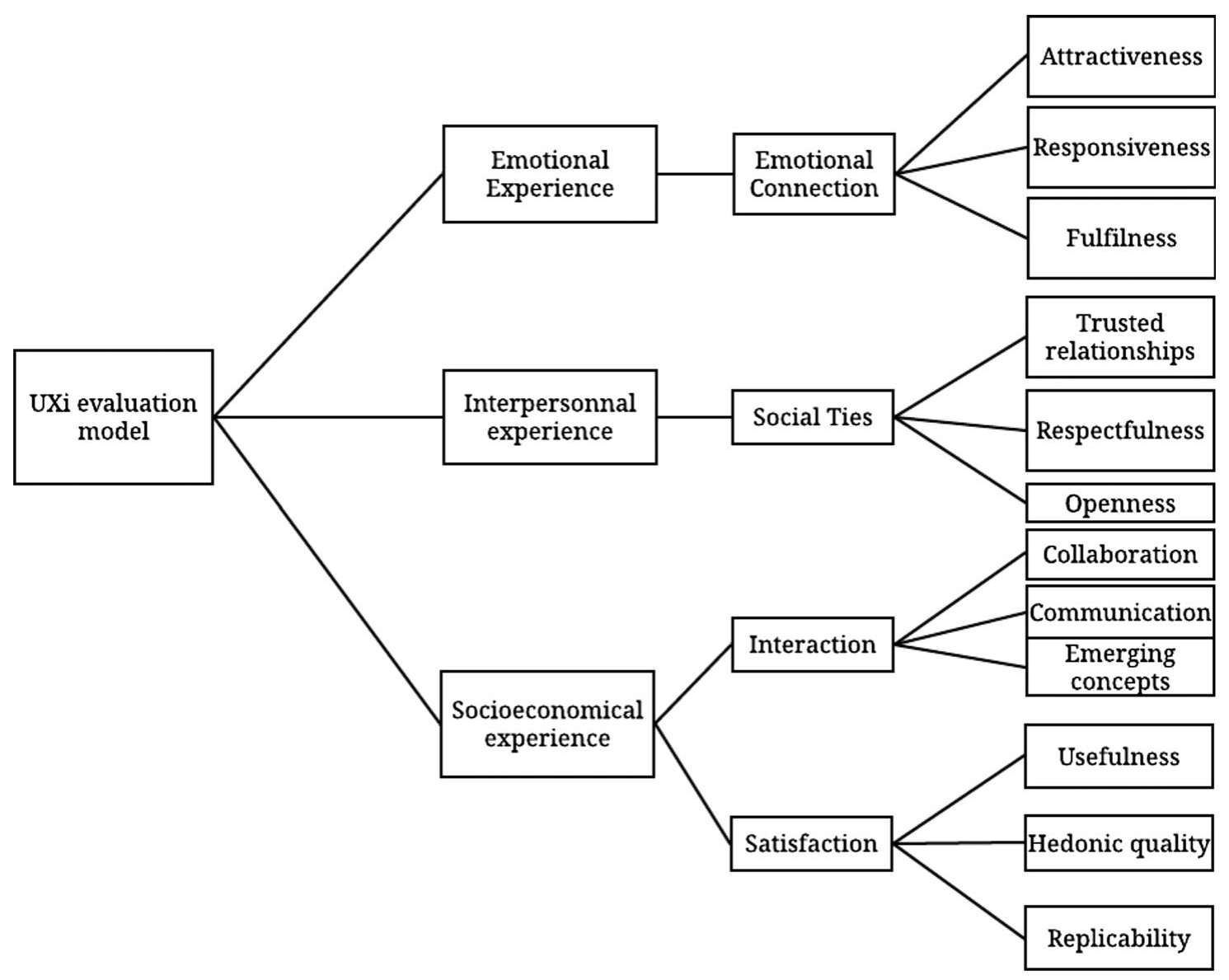

Fig. 2. UXi construct (Lecossier et al., 2018a, in press).

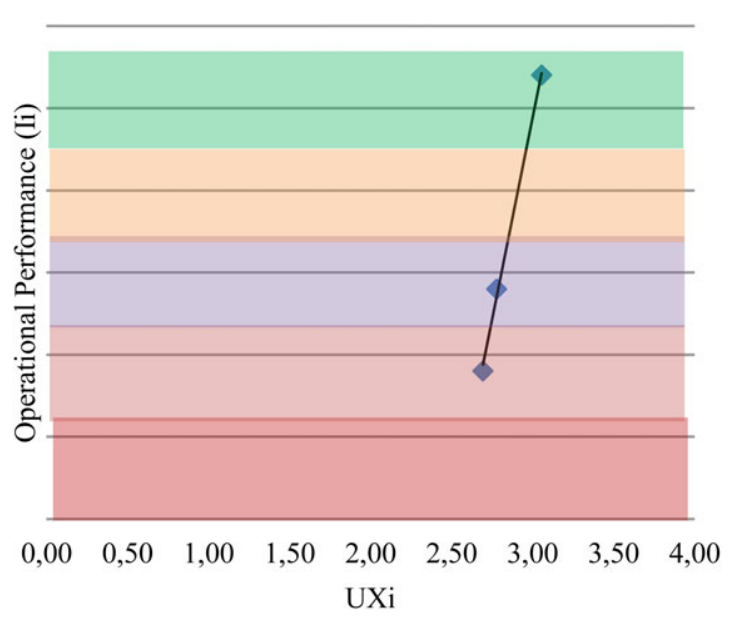

Fig. 3. Relationship between li and UXi (real and hypothetical) for radical innovation projects with $\mathrm{Ci}=$ constant.

process and the ideation stage operate and to describe the expected goals for all project team members. As a follow-up, other sessions were regularly organized to support the teams in the construction of their innovative concepts. Sessions were held on the working time of employees.

For all three projects, UXi assessments of co-creator groups and project operational performance were completed at least 6 months after the first session of the ideation stage. A jury at an innovation committee made the evaluation of the operational performance of projects A and B. It is during this committee, open to all employees of the company, that the members of the innovation projects gave a presentation of three innovative concepts per project. The jury then assessed the maturity of the proposed concepts using the SAPIGE grid and asked questions to the project leaders. The evaluation of the operational and innovation performance of Project $\mathrm{C}$ was carried out by a group of people including co-creators of the project. The only concept proposed for Project C was also evaluated using the SAPIGE grid.

The evaluation of the UXi, was carried out in using a questionnaire that was given to the co-creators of the three projects two days after the innovation committee for projects $\mathrm{A}$ and $\mathrm{B}$, and after the group evaluation for project $\mathrm{C}$.

\section{Hypothesis}

In order to meet the objective (2), we make the following hypotheses:

- Hn: The operational performance of a radical innovation project of our pilot company depends on the $\mathrm{H} 1 / \mathrm{H} 2 / \mathrm{H} 3 / \mathrm{H} 4$ experience of its group of co-creators.

o H1: socio-economic;

○ $\mathrm{H} 2$ : interpersonal; 
○ H3: emotional;

○ H4: user UXi (combines H1, H2, and H3).

\section{Participants}

In total, 35 individuals participated in this study. Most of them were employees of our pilot company with different profiles of co-creators who participated voluntarily in radical innovation projects. Similarly, the evaluators who participated in the innovation committee, for the evaluation of projects $\mathrm{A}$ and $\mathrm{B}$ and the evaluation of project $\mathrm{C}$, had complementary profiles.

\section{Methodology}

As the first step, we perform a descriptive analysis of the quantitative and qualitative results of UXi evaluations of co-creator groups. We are then able to identify the operational elements that impact the UXi, its three types of experience and its 12 properties from the point of view of the co-creators of our pilot company.

In the second step, the quantitative results of the performance evaluations of the three projects are represented through the use of graphs and histograms. We then perform a descriptive summary of the operational performance of each project by combining the quantitative results of the ratings with the resource expenditures.

Finally, we observe, through a new descriptive analysis, dependencies between the operational performance of innovation projects and the UXi of co-creator groups. We crosscheck for this on the results of the performance of innovation projects with the qualitative and quantitative results of the UXi co-creator groups. To conclude, we look at the possibility to validate the hypotheses with a qualitative approach.

\section{Evaluation instrument}

\section{UXi evaluation}

We have designed a mixed methods instrument through a bipolar survey questionnaire that includes 12 items representative of the 12 properties of the reorganized model of UXi. While the wording of the questions has been adapted to the context of the company, all items remain the same. The resulting instrument is a bipolar survey that incorporates an open question for each bipolar question so that respondents can mention the reasons and motivations for justifying their scores.

\section{Operational performance evaluation}

As we have seen, the evaluation of the operational performance $\mathrm{Pi}$ of a radical innovation project is based on two aspects of which we detail here the means of evaluation:

1 - Evaluate the evidence that the project is useful and innovative: for this evaluation we chose to use the SAPIGE grid because it is generic and therefore adaptable to radical innovation projects of different typologies (eg product, organization). In this grid, each piece of evidence (eg expression of need, relevance of use, integration of constraints) is evaluated using a bipolar scale (0: no evidence; 1: some evidence; 2: credible evidence, 3 : credible and indisputable evidence) that incorporates an open question allowing evaluators to justify reasons and motivations on each of their ratings. Evaluators' scores are averaged to give an overall score to an item of evidence. When a project proposes several concepts, the scores of the same evidence element of the concepts are averaged to give the score of the evidence element of the innovative project.

2 - Evaluate the resources spent to gather these evidences: for this evaluation we chose to quantify the resources spent for each project during the 6 months of the ideation phase. In accordance with the indicators used in the project management processes of our pilot company, we evaluated the expenditures of the three radical innovation projects according to the following indicators: number of hours, budget, and deadlines.

\section{Data collection}

Collecting data on UXi

For all three projects, respondents $(N=23)$ completed the corresponding questionnaire including:

(a) Quantitative evaluation through the use of a bipolar evaluation based on a semantic scale. For example: 0: Useless; 1: Mostly Useless; 2: Almost Useful; 3: Useful; 4: Very Useful.

(b) A qualitative assessment through an open question to provide a justification in terms of reasons and motivations for each rating.

\section{Collecting data on operational performance}

- Projects A and B:

The members of the juries $(n=6$ and $n=5)$ completed the SAPIGE evaluation grid on several occasions, including:

(a) A quantitative evaluation of the evidence through the use of an assessment based on a bipolar scale.

(b) A qualitative assessment through an open question to provide a justification in terms of reasons and motivations for each rating.

In total, the A jury members completed three evaluation grids for the three concepts of the innovative project A. The B jury members completed three evaluation grids for the three concepts of the innovative B project.

- Project C:

By interacting with each other, Project C evaluators $(n=11)$ individually completed the SAPIGE evaluation grid including:

(a) A quantitative evaluation of the evidence through the use of an assessment based on a bipolar scale.

(b) A qualitative assessment through an open question to provide a justification in terms of reasons and motivations for each rating.

In total, the evaluators completed only one evaluation grid to evaluate the single concept of project C.

\section{Sampling}

\section{Sampling and processing of UXi data}

Using the same method as in the preliminary experiment for validating the model, we compare the qualitative and quantitative data in order to ensure that respondents correctly interpreted all questions.

Figure 4 shows the results of this comparison. On the $x$-axis, there are 12 properties of the UXi evaluation model that were evaluated by 12 bipolar questions. Along the ordinates, there is the rating scale from 0 to 4 . The first level from 0 to 1 is considered "unsatisfactory"; the second level from 1 to 2 is described as 


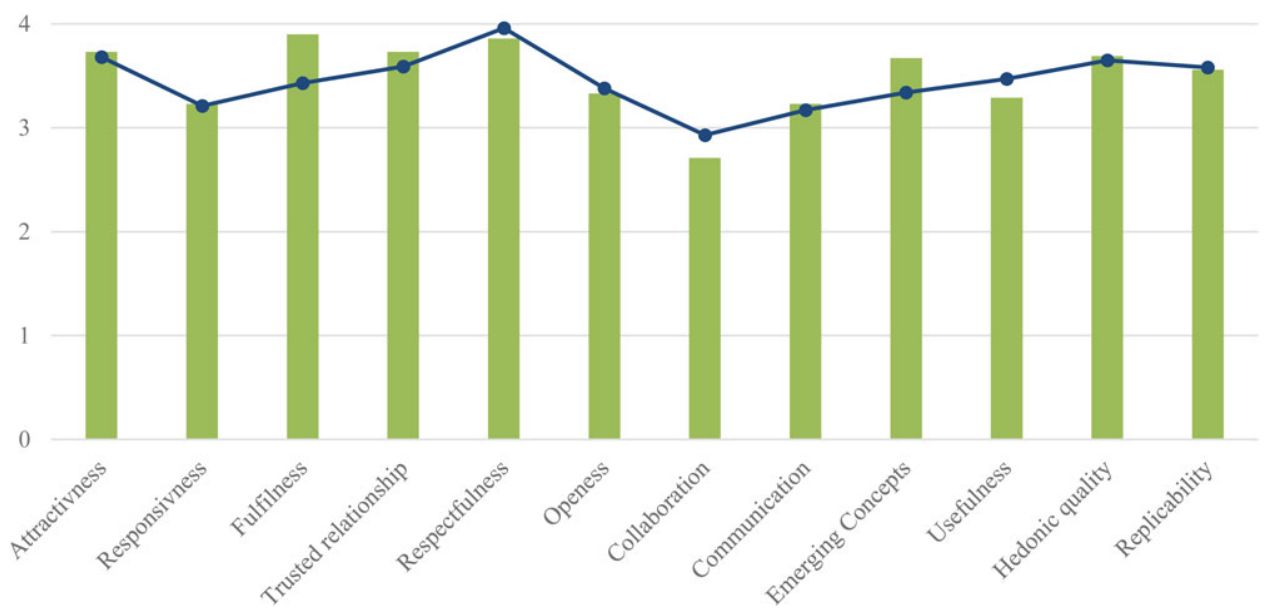

Fig. 4. Comparison of quantitative and qualitative data for all 12 properties of UXi ( $N=23$ respondents).

"rather unsatisfactory"; the third level from 2 to 3 is considered "almost satisfactory"; finally, the fourth level is qualified as "satisfactory".

The same axis is also used to represent negative comments (value 0 ) to $\mathrm{Y}$ : 0 , positive comments (value 1 ) to $\mathrm{Y}$ : 4 , as well as negative/positive comments that cancel each other out (value $0.5)$ to $\mathrm{Y}: 2$.

The averages of the negative and positive comments given for each evaluation appear as a bar graph while the averages of the bipolar notation for each property of the UXi appear as a point on a curve.

None of the results of the qualitative evaluation shows a contrast with the quantitative evaluation. This means that the visible difference between the two ratings for the properties "Excitement" and "Emergence of concepts" does not question the nature of the results (unsatisfactory vs. satisfactory). The data from the 12 items can therefore be used for the rest of our study.

\section{Sampling and processing of operational performance data}

\section{- Projects A and B:}

Projects A and B have three concepts each: concepts A01, A02, and $\mathrm{A} 03$ for project $\mathrm{A}$; concepts $\mathrm{B} 01, \mathrm{~B} 02$, and $\mathrm{B} 03$ for project $\mathrm{B}$.

The evaluation of a project is equal to the average of the evaluations of the concepts. As a result, the Project A score is equal to the average score of A01, A02, and A03, while the Project B score is equal to the average score of B01, B02, and B03. Each score of a concept is equal to the average score of the members of the jury.

- Project C:

Project $\mathrm{C}$ has got only one concept that is evaluated by the average of the scores of the 11 evaluators.

\section{UXi results}

\section{At the level of experience type}

The evaluation of UXi shows us that the three types of experience were well evaluated for the three projects A, B and C. The already analyzed qualitative comments help to understand the scores of the UXi properties and also allow us to describe more precisely the results at the level of experience types.

- Emotional experience:

Obtained results show that the co-creators of the three project teams experienced a pretty good emotional experience through their involvement in these innovation projects (Fig. 5). This is logical since the emotional experience is composed of the properties "Excitement", "Engagement", and "Attractiveness" that have got a high score. Thus, one can argue that the organizational elements listed in Table 3 enabled the co-creators of the three innovation projects to have an average emotional experience of 3.43 score on a scale ranging from a minimum of 0 up to a maximum of 4 .

- Interpersonal experience:

Again, results show that the co-creators of the three project teams had a pretty good interpersonal experience through their involvement in these innovation projects (Fig. 5). This observation makes sense since the interpersonal experience consists of the properties "mutual respect", "mutual trust", and "openmindedness" equally well evaluated. Thus, we could argue that the organizational elements listed in Table 4 enabled the co-creators of the three innovation projects to have an average interpersonal experience of 3.68 on a maximum of 4 .

- Socio-economic experience:

The co-creators of the three project teams had a pretty good socio-economic experience through their involvement in these innovation projects (Fig. 5). However, there is a larger rating gap across projects for the socio-economic experience since it consists of the four properties for which a qualitatively significant difference has been identified. As a reminder, this difference is mainly due to the fact that the co-creators of projects A and B respectively expressed the following comments: "not having enough time to see each other, not having been able to carry out creative activities, having encountered difficulties with complex activities to apprehend."

Therefore, we can argue that the organizational elements listed in Table 5 enabled the co-creators of the three innovation projects to have an average socio-economic experience scoring slightly below the two previous types of experience with an average score of 3.37 .

\section{At the level of UXi}

Figure 6 shows that the UXi score of the three project co-creators is relatively close. Thought, the UXi score of the project A co-creators is lower than the one of project $\mathrm{B}$ co-creators, which is itself lower than the score of the project $\mathrm{C}$ co-creators. Nevertheless, it is difficult to draw a conclusion on this outcome because the evaluation of the UXi of the project $\mathrm{C}$ co-creators was 


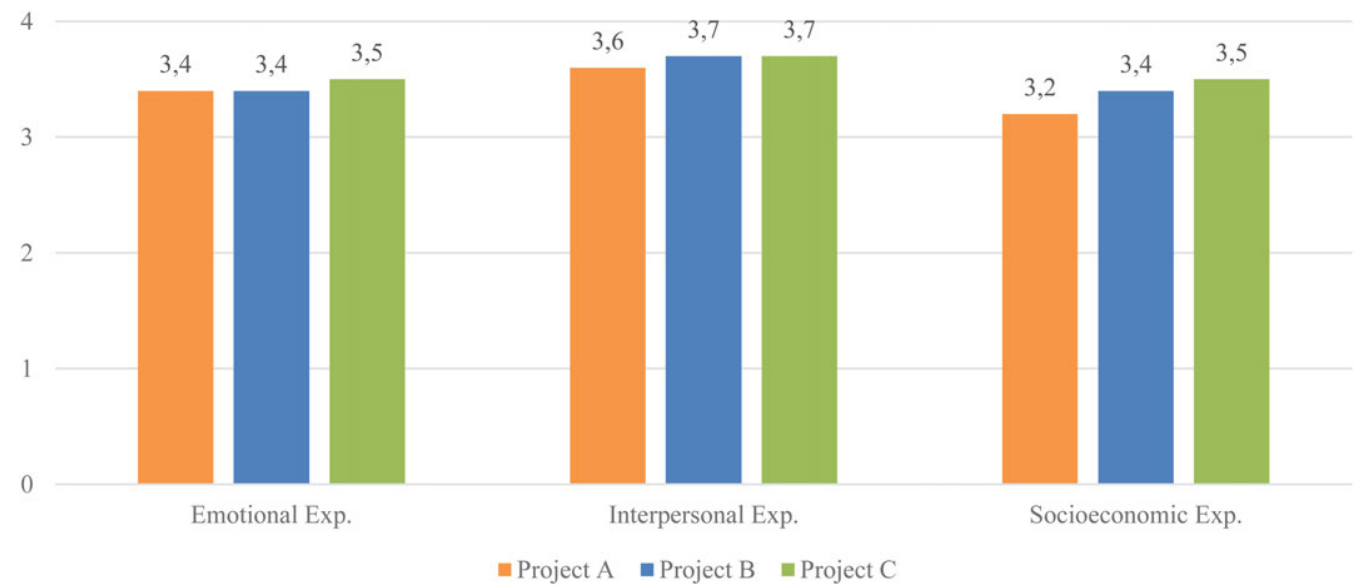

Fig. 5. UXi Quantitative Results of Co-Creator Groups of Projects A, B, and C at the Level of Experience Types.

Table 3. Organizational elements that positively impact the emotional experience

- Work on a new and interesting subject that provokes the feeling of being a player in an emerging or future change.

- Have the desire to do a collective job.

- Participate on a voluntary basis.

- Work with people they do not know much about.

- Have the feeling of autonomy and freedom of action.

- Work in a new way and unconventional organization where the animation methods avoid boredom and make it possible to get out of everyday life.

- To be accompanied by an animation that optimizes the work and desire for collective fulfillment.

Table 4. Organizational elements that positively impact the interpersonal experience

- Work in a group where hierarchy levels have been switched-off.

- Remove all bad habits from the start of the project.

- Have a rule of the game that explains the need and the legitimacy to remain open while taking time to be creative.

- Listen to each other.

- Have the confidence and freedom to express whatever you wish to.

- Undertake collective decisions.

o Work in a friendly atmosphere.

not carried out under the same conditions as those of the co-creators' UXi of the projects A and B. However, the analyses carried out at the level of properties and types of experience have shown that the socio-economic experience of the co-creators was lower for Project A. This was partly due to some co-creators reporting that they did not have enough opportunity to perform Creativity activities, having difficulties in apprehending certain methods, thinking that even without the innovation project the subject treated would continue to advance.

Ultimately, the overall analysis of UXi's properties and types of experience allows us to argue that the UXi of the three innovation project co-creators conducted within our pilot company is rather positive. The first part of our experimentation demonstrates that the UXi of the co-creators of our pilot company is positively impacted by the fact that they can carry out the activities listed in Table 6.
Table 5. Organizational elements that positively impact the socio-economic experience

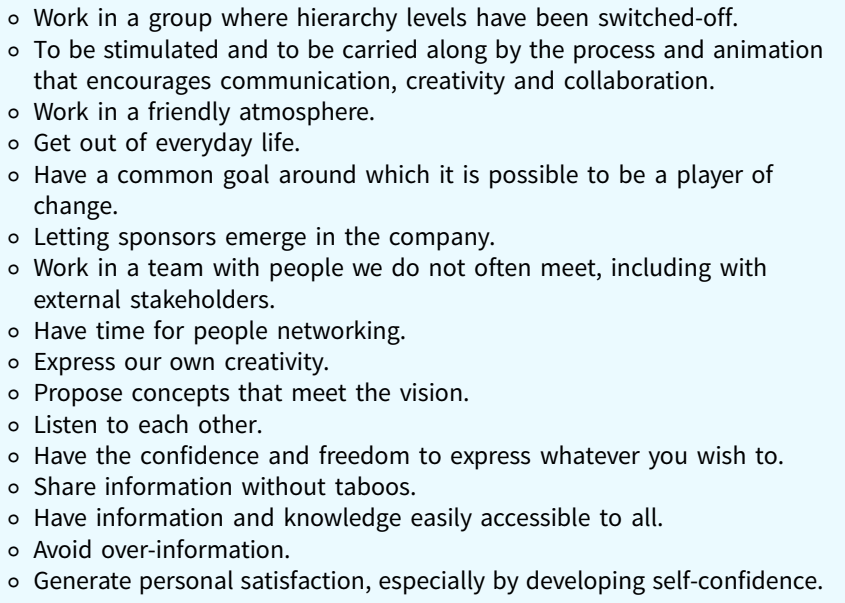

\section{Performance of radical innovation projects}

The performance of innovation projects $(\mathrm{Pi})$ is evaluated on two aspects: (1) evaluation of evidences showing that the project is useful and innovative; (2) evaluation of the resources spent to gather these evidences. Therefore, we propose a report of the evaluations of the evidences of the three innovative projects. Then, we summarize the resources spent on each project. Finally, we perform a descriptive analysis of the performance of radical innovation projects by combining the spent resources with the results of the evidence evaluations.

Table 7 summarizes the performance of the three radical innovation projects conducted in the context of our pilot company. It appears that the results are fairly consistent both in terms of resource consumption and participation rate. Furthermore, we observe that the proof of utility score is higher for the Project $\mathrm{C}$ (i.e. 2.10). We do believe that this difference is most probably due to the fact that the co-creators of project $\mathrm{C}$ proposed a single concept on which the resources were focused.

The scores of Project A innovation and utility evidence are lower than Project B scores, while three concepts were proposed for each project. This discrepancy is likely to come from the 


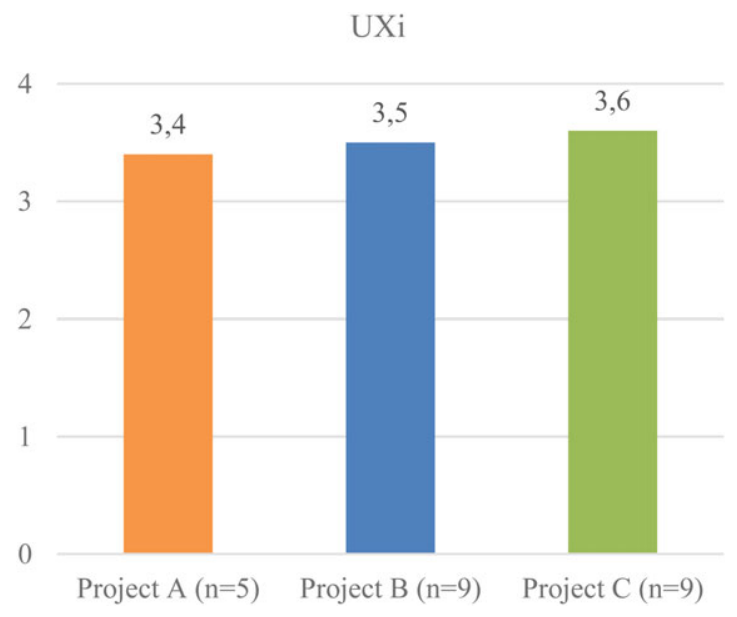

Fig. 6. Quantitative UXi results of projects A, B, and C co-creators.

Table 6. Organizational elements that positively impact the UXi

- Work on a new and interesting subject that provokes the feeling of being an actor of an emerging or future change and gives the desire to accomplish a collective work.

- Participate on a voluntary basis and have the feeling of autonomy and freedom of action.

- Work in a new way in a new, friendly, and unconventional organization where the animation methods avoid boredom and make it possible to get out of everyday life.

- Work in a team with no hierarchical level, with people who do not get along very often including external stakeholders while being able to undertake collective decisions.

- To be stimulated and to be carried along by the process and an animation that encourages communication, creativity and collaboration while optimizing the work and desire for collective fulfillment.

- Have a rule of the game that explains, from the start of the project, the need and the legitimacy to remain open, take the time to be creative, remove all negative habits and take time for people networking.

- Listen to each other, have the confidence and freedom to express whatever, share information without taboos and have easy access to information and knowledge without being over-informed.

- Be personally satisfied by proposing concepts that meet the vision while letting emerge sponsors in the company and developing self-confidence.

more complex typology of Project A, which aims to create a breakthrough innovation. Finally, the Project B aims to create an organizational breakthrough innovation for which data are easier to obtain since they are most often found within the organization.

\section{Links between Pi and UXi}

In this section we present the outcome concerning the objective (2) of this experimentation as explained above. It consists in verifying the existence of links between the UXi, its types of experience and the operational performance of an innovation project. Table 7 presents a summary of information relating to the three radical innovation projects within the context of our pilot enterprise.

Considering the limited number of projects and the minor differences between the operational results of each project, it would be doubtful to perform some statistical analysis in order to check the influence of one parameter on another. Hence, we are fully satisfied with a descriptive analysis of these data. Based on the
Table 7. Operational performance of Projects A, B and C

\begin{tabular}{lccc}
\hline & Project A & Project B & Project C \\
\hline Utility proof & 1.60 & 1.89 & 2.10 \\
\hline Innovation proof & 1.65 & 1.96 & 1.67 \\
\hline Problematic definition & 1.80 & 2.17 & 2.90 \\
\hline Expression of need & 1.73 & 2.06 & 2.40 \\
\hline Users identification & 1.33 & 1.67 & 1.40 \\
\hline Relevance of use & 1.80 & 2.06 & 1.78 \\
\hline Constraints integration & 1.33 & 1.50 & 2.00 \\
\hline Knowledge of the value chain & 1.80 & 1.89 & 2.00 \\
\hline Realization of a legal watch & 1.13 & 1.83 & 0.5 \\
\hline Benchmarking & 1.73 & 2.06 & 1.89 \\
\hline Valuation of the project's & 1.93 & 2.06 & 2.30 \\
\hline strengths & 216 & 190 & 195 \\
\hline Financial expenses (k€) & 20 & 0.2 & 0.5 \\
\hline Number of sessions & 20 & 23 \\
\hline Hourly expenses (hours) & & & \\
\hline
\end{tabular}

above results, it appears that the socio-economic experience depends on the capacity of an organization to help its co-creators in: (1) having a common goal around which it is possible to be a player of change; (2) proposing concepts that meet a vision; (3) facilitating creativity; (4) letting emerge sponsors within the organization. Therefore, if the socio-economic experience is satisfying, it means that the company's innovation process allows co-creators to have the vision to rely on in order to identify usefulness and innovativeness evidences.

Indeed, it is likely that an organization facilitating its co-creators to: (1) get out of everyday boredom; (2) find time to see each other; (3) work in a team with people they do not see much, including with external stakeholders, allow to better identify users and needs, define the value chain, and perform a technical and economic watch.

Satisfying socio-economic experience also depends on the organization's ability to stimulate and support its co-creators through a process and animation that encourages communication, creativity, and collaboration. According to the following provided feedback: "The pace of advancement was sustained leaving little room to boredom; The organization of the sessions was excellent.", a group of co-creators who no longer have to think about how to organize themselves for innovating will be more effective.

It also appears that the interpersonal experience depends on: (1) the ability of an organization to switch-off hierarchical levels and the bad habits that co-creators usually have with each other from the start of the project; (2) facilitating collective decisionmaking; (3) listening and freedom of expression; (4) making it legitimate to stay open and take the time to be creative. Encouraged by these organizational elements, a satisfying interpersonal experience helps strengthen the social bonds between the members of a group in order to improve the capacity for interaction among them. Therefore, it allows the group of co-creators to better perform together.

According to the following provided feedback: "All members of the team were on the same wavelength"; "Listening to each other uninhibited", allowing a group of co-creators to have a 
satisfying interpersonal experience is therefore a lever to improve the socio-economic experience and thus be more effective in identifying usefulness and innovativeness evidence.

As earlier revealed, the emotional experience depends on the ability of an organization to: (1) convey willingness to its co-creators to volunteer on collective work; (2) offer autonomy and freedom of action; (3) provide the means to work from the unconventional way in order to avoid boredom. Thus, a satisfying emotional experience is a lever of action that relies on excitement and attractiveness to maintain the commitment of co-creators along their project.

According to the following provided feedback: "We feel more engaged"; "However, we must succeed in freeing ourselves in our respective schedules inevitably loaded and monopolized by our daily duties.", a satisfying emotional experience appears to be fundamental since it is the reason why the co-creators remain engaged over a period of 6 months; despite the difficulties they were facing for having time to regularly meet for completing their innovation project.

Ultimately, the UXi depends on the ability of an organization to support its groups of co-creators in the ideation activities of their radical innovation projects, with the right balance between structuring and freedom. Therefore a satisfying UXi allows the co-creators to have a satisfying emotional experience in order to make sure they engage in their projects; as well as a satisfying interpersonal experience in order to make sure they collaborate properly; and a satisfying social experience in order to ensure that they adequately perform in building their innovation projects. Finally, we can therefore argue that the performance of an innovation project depends on its co-creators' UXi.

\section{Discussion and findings}

This experimentation was carried out on three radical innovation projects taking place within our pilot company. First of all, it consisted in identifying the operational and organizational elements that impact the UXi of the co-creators. Then, the purpose of this experimentation was to verify the existence of dependencies between the UXi of co-creator groups and the operational performance of their innovation projects.

\section{Elements that impact the UXi of co-creator groups}

The initial results allow identifying the organizational elements that impact the properties and types of UXi and UXi experiences within our pilot enterprise (Tables 3-6). For example: (1) working in a group where the hierarchical levels have been switched-off and in a friendly atmosphere; (2) out of daily routine and duties; (3) participate voluntarily and have the feeling of autonomy and freedom of action. These results are supported by previous studies on the culture of innovation confirming that it is necessary, among other things, to: (1) foster freedom; (2) trust groups that innovate; (3) develop trust in these groups (McLaughlin et al., 2008).

Other elements that impact UXi have been identified along our experimentation and highlight the interest in UXi and its evaluation. For example, this is the case for the contradiction between the needs of co-creators willing to be stimulated by the process and an animation that encourages communication, creativity, and collaboration, while participating on the basis of volunteering and having the feeling of autonomy and freedom of action. These results also provide a complementary view to the work of Leifer et al. (2001), who advocate minimally building a pole of radical
Table 8. Summary of project information

\begin{tabular}{lccc}
\hline & Project A & Project B & Project C \\
\hline UXi & 3.4 & 3.5 & 3.6 \\
\hline Emotional experience & 3.4 & 3.4 & 3.5 \\
\hline Interpersonal experience & 3.6 & 3.7 & 3.7 \\
\hline Socio-economic experience & 3.2 & 3.4 & 3.5 \\
\hline li & 1.63 & 1.92 & 1.88 \\
\hline Utility proof & 1.60 & 1.89 & 2.10 \\
\hline Innovation proof & 1.65 & 1.96 & 1.67 \\
\hline Financial expenses (k€) & 2 & 0.2 & 0.5 \\
\hline Number of sessions & 20 & 20 & 23 \\
\hline Hourly expenses (hours) & 216 & 190 & 195 \\
\hline Duration (months) & 6 & 6 & 6 \\
\hline
\end{tabular}

innovation to innovate in a mature company. They argue that an innovation pole can supervise and help developing projects by reducing uncertainty without increasing bureaucracy burden.

\section{Dependance between the UXi and the operationnal performance}

Our first hypothesis indicated that the operational performance of a radical innovation project depended on the socio-economic experience of the group of co-creators. This hypothesis is partially validated. Indeed, the socio-economic experience depends on the ability of an organization to help its co-creators to have a common goal around which it is possible to be an actor of change, to propose concepts that meet a vision, to enable creativity, to let emerge sponsors within the company (Table 5). In our experiment, the socioeconomic experience averaged 3.37 on a maximum of 4 . This satisfying experience allowed, over a period of 6 months, the co-creators of the three innovation projects to gather evidence of usefulness on average equal to 1.86 on a scale from 0 to 3 and close to a score of 2 "credible elements", and evidence of innovation averaging 1.76 on the same scale. Thus, if the co-creators rated this socio-economic experience as unsatisfying, then, the performance of their project would most probably be lower as a direct consequence.

Our second hypothesis suggested that the operational performance of a radical innovation project of our pilot company depended on the interpersonal experience of the group of co-creators. This hypothesis is also partially validated. Indeed, interpersonal experience can be seen to impact the operational performance of a radical innovation project through socio-economic experience by strengthening the social bonds of a group of co-creators. This includes switching-off hierarchical levels and minimizing bureaucracy burden within co-creator groups (Table 4). These results are in line with previous studies conducted on the culture of innovation (Ahmed, 1998; Koen et al., 2001).

Our third hypothesis stated that the operational performance of a radical innovation project of our pilot company depended on the emotional experience of the group of co-creators. It is also partially validated. Indeed, the results show that the emotional experience does not directly impact the performance of the radical innovation project but rather the other types of experiences. However, the emotional experience is one that maintains a good level of commitment and engagement of co-creators to their projects (Table 3 ). 


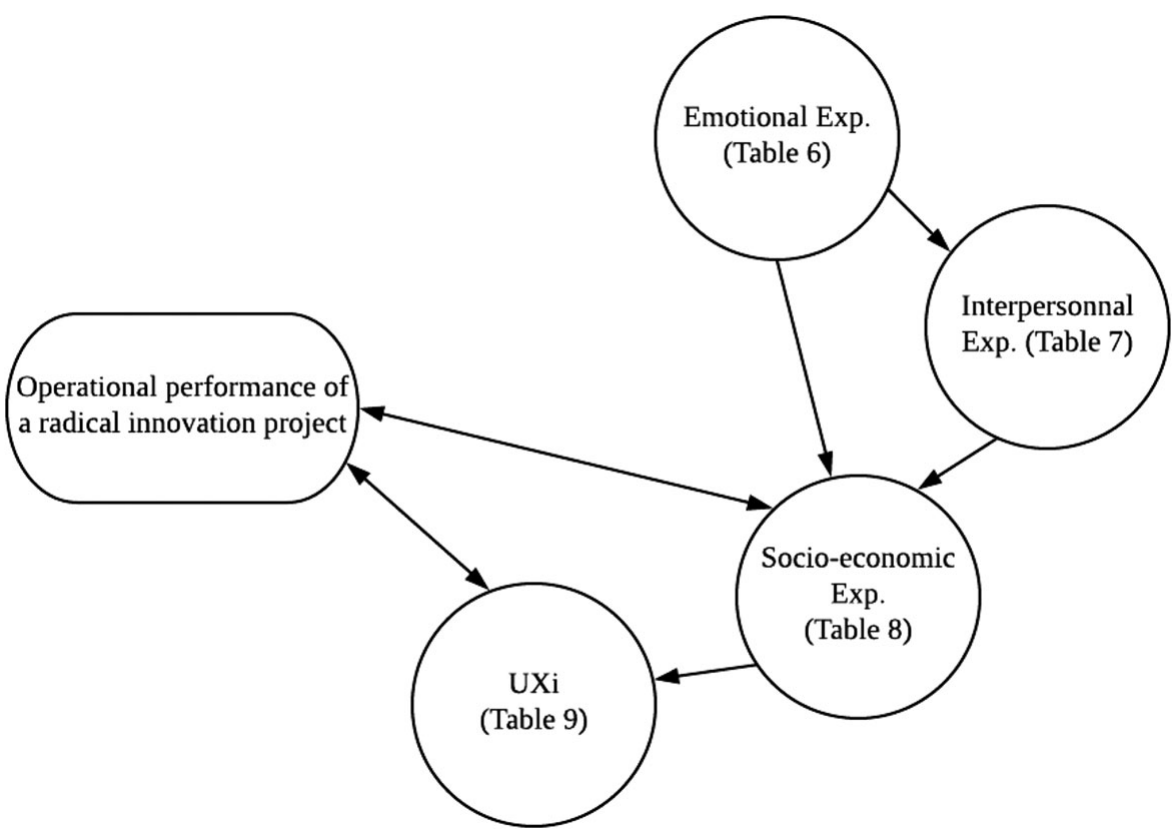

Fig. 7. Representation of the dependencies between UXi, its types of experience and the operational performance of a radical innovation project in our pilot company.
This emotional experience is essential for radical innovation projects for which the ideation activities lasts at least 6 months like in our pilot company. These results are in line with the previous study stating that committed individuals are more inclined to develop long-term relationships with their projects (Dessart et al., 2015). They also support the idea that the ideation stage of the upstream innovation process should allow co-creators to live an exciting, engaging and attractive experience.

Our fourth and last hypothesis suggested that the operational performance of a radical innovation project of our pilot company depended on the UXi of the co-creators group. It is validated. Indeed, UXi is composed of emotional, interpersonal, and socioeconomic experiences; therefore, impacts the operational performance of a radical innovation project.

\section{Limitations}

The first limitation of this study concerns first the number of limited projects $(N=3)$. Even if the analysis of three projects for such a large study can be considered sufficient, we would have preferred to have more data in particular in order to quantitatively verify the research hypotheses.

The second limitation concerns the homogeneity of UXi results of co-creators and the operational performance of projects (Table 8). Indeed, the differences between the projects being weak it is difficult to draw conclusions. On the other hand, the qualitative analysis made it possible to explain precisely the results of the evaluations and to establish representative observations of the real facts.

\section{Conclusion and future work}

The results of this experiment allowed us to better understand the elements that positively impact UXi and its types of experience within our pilot company (Tables 3-6). Overall, the elements that impact UXi in our pilot company are the hallmarks of a satisfying culture of innovation (Ahmed, 1998; Padilha and Gomes, 2016). This shows that UXi is well representative of the social aspect intrinsic to innovation.
In addition, the above-described results also showed us that adequate support to co-creators is essential for being able to innovate radically in our pilot company. Indeed, the established codes are too heavy to be overtaken by simple instructions of freedom. It is then necessary to define operating rules to support collective decision-making or to legitimize entrepreneurial behavior from the start of the project.

Interestingly, we learnt that it is possible to easily assess the organization's ability to properly take into account the intrinsic social aspect of innovation. This assessment can be done simply by sending a questionnaire based on the UXi to the co-creators of innovative projects; hence, not necessarily requiring a much more demanding organizational diagnosis.

Finally, this experiment results enabled us to qualitatively validate the dependencies between the UXi of co-creator groups and the operational performance of innovation projects. Figure 7 outlines the system of dependency relationships between UXi, its types of experience, and the operational performance of a radical innovation project.

In terms of perspective, we think it would be good to conduct UXi evaluations of groups of co-creators working on radical innovation projects in companies similar to our pilot company in order to acquire more data on the subject. This would allow quantitative and statistical analysis for verifying the veracity of the dependencies between UXi, its experience types and the operational performance of a radical innovation project as shown in Figure 7.

Acknowledgements. This empirical study was partly funded by the company SOURIAU ESTERLINE. Authors wish to acknowledge the support, openness, and willingness of SOURIAU ESTERLINE to explore a new approach for generating radical innovations.

\section{References}

Ahmed PK (1998) Culture and climate for innovation. European Journal of Innovation Management 1, 30-43. Available at http://www.emeraldinsight. com/doi/abs/10.1108/14601069810199131.

Baregheh A, Rowley J, Sambrook S (2009) Towards a multidisciplinary definition of innovation. Management Decision 47, 1323-1339. 
Christensen C (1997) The Innovator's Dilemma. Boston: Harvard Business School Press.

Cooper R (2008) Perspective: the stage-gate idea to launch process - update, what's new and nexgen systems. Journal of Product Innovation Management 25, 213-232. Available at http://www.stage-gate.com/downloads/wp/wp_30.pdf.

Cooper RG (2001) Winning at New Products: Accelerating the Process From Idea to Launch, Cambridge: Third Edition Basic Books, 2001. Cambridge: Third edit. B. Books, ed.. Available at http://inkinghub.elsevier.com/ retrieve/pii/0024630189901854\%5Cn; http://www.jstor.org/stable/10.2307/ 2393871\%5Cn; http://search.ebscohost.com/login.aspx?direct=true\&db= bth\&AN=6538249\&site $=$ ehost-live.

Cresswell JW, Plano Clark VL, Gutmann ML and Hanson WE. (2003) Advanced mixed methods research designs. In A. Tashakkori \& C. Teddlie (Eds.), Handbook of mixed methods in social and behavioral research (pp. 209-240). Thousand Oaks, CA: Sage.

Dale L (2015) The Experience Makes the Product, Not the Features: A Comprehensive Guide for Using your MVP to Get the right Product to Market, Fast. UX Magazine.

De Brentani U and Reid SE (2012) The fuzzy front-end of discontinuous innovation: insights for research and management. Journal of Product Innovation Management 29, 70-87.

Dessart L, Veloutsou C and Morgan-Thomas A (2015) Consumer engagement in online brand communities: a social media perspective. Journal of Product \& Brand Management 24, 28-42. Available at http://www. emeraldinsight.com/doi/10.1108/JPBM-06-2014-0635.

E.Porter M (1985) Competitive Advantage - Creating and Sustaining Superior Performance (The Free P). New York, NY, US.

Frishammar J, Floren $\mathbf{H}$ and Wincent J (2011) Beyond managing uncertainty: insights from studying equivocality in the fuzzy front End of product and process innovation projects. Ieee Transactions on Engineering Management 58, 551-563.

Gaia R and Kirca AH (2012) Firm innovativeness and its performance outcomes: a meta-analytic review and theoretical integration. Journal of Marketing 76, 130-147. Available at https://doi.org/10.1509/jm.10.0494.

Garcia R and Calantone R (2002) A critical look at technological innovation typology and innovativeness: a literature review. The Journal of Product Innovation Management 19, 110-132.

Hart T, Jacobs P and Mangqalaza H (2012) Key concepts in Innovation Studies. Towards working definitions. (December 2012). Available at http:// www.hsrc.ac.za/uploads/pageContent/5092/RIAT2012_Innovationworking Definitions_ConPaper2.pdf.

Hart TGB, et al. (2015) Revealing the social face of innovation. South African Journal of Science 111, 109-114.

ISO FDIS 9241-210 (2009) Ergonomics of human-system interaction - Part 210: Human-centered design for interactive systems (formerly known as 13407). International Organization for Standardization (ISO). Switzerland.

Ind N and Coates N (2013) The meanings of co-creation. European Business Review 25, 86-95.

Kim J and Wilemon D (2002) Focusing the fuzzy front-end in new product development. R\&D Management 32, 269-279. Available at http://doi. wiley.com/10.1111/1467-9310.00259.

Koen P, et al. (2001) Providing clarity and a common language to the "fuzzy front end". Research Technology Management 44, 46-55.

Koen PA et al. (2002) Fuzzy front end: effective methods, tools, and techniques. In Paul Belliveau and Abbie Griffin and Stephen Somermeyer (Eds.), PDMA Toolbook for New Product Development. New York, NY: John Wiley and Sons, pp. 5-35.

Krawczyk P, Topolewski M and Pallot M (2017) Towards a Reliable and Valid Mixed Methods Instrument in User eXperience Studies. In International Conference on Engineering, Technology and Innovation (ICE/ITMC) (pp. 1497-1506). Funchal: IEEE Technology and Engineering Management Society (TEMS).

Law EL-C et al. (2009) Understanding, scoping and defining user experience. Proceedings of the 27th International Conference on Human factors in computing systems - CHI 09, (April 2016), p. 719. Available at http://dl.acm.org/ citation.cfm?doid=1518701.1518813.

Lecossier A and Pallot M (2017) UX-FFE Model: An Experimentation of a new innovation process dedicated to a mature industrial company. In 23rd International Conference on Engineering, Technology and Innovation (ICE-IEEE-TEMS 2017). Funchal.

Lecossier A et al. (2016) Une vision multidimensionnelle des typologies d'innovation pour identifier et concevoir une démarche d'innovation. In CONFERE 16. Prague. Available at http://sam.ensam.eu/handle/10985/ 11148 (Accessed 27 September 2016).

Lecossier A, Pallot M et al. (2017a) Modèle UX-FFE : expérimentation de la phase de validation d'un nouveau processus d'innovation dédié a une entreprise industrielle mature. In CONFERE 17. Séville.

Lecossier A, Tcha-Tockey $\mathbf{K}$ and Richir S (2017b) The user experience measurement: a challenge of the twenty-first century. In International Scientific Conference dedicated to the 175th Anniversary of the D.I. Mendeleyev Institute for Metrology (VNIIM 175). Saint-Petersburg: Book of Abstracts.

Lecossier A et al. (2018a) Application of the UX-FFE model for optimizing the performance of the upstream innovation process. In $24 \mathrm{rd}$ International Conference on Engineering, Technology and Innovation (ICE-IEEE-TEMS 2018). Stuttgart.

Lecossier A, Pallot M, Crubleau $\mathbf{P}$ and Richir $\mathbf{S}$ (in press) Construction of an instrument to evaluate the User eXperience of a group of co-creators in the upstream innovation process. International Journal of Services Operations and Informatics.

Leifer $\mathbf{R}$ et al. (2001) Implementing radical innovation in mature firms: the role of hubs. Source: The Academy of Management Executive 15, 102113. Available at http://www.jstor.org/stable/4165764\%0A; http://www. jstor.org/stable/4165764?seq=1\&cid=pdf-reference\#references_tab_contents \%0A; http://about.jstor.org/terms.

Lucas H and Goh JM (2009) Journal of strategic information systems disruptive technology: how kodak missed the digital photography revolution. Journal of Strategic Information Systems 18, 46-55. Available at http://dx. doi.org/10.1016/j.jsis.2009.01.002.

Markham SK (2013) The impact of front-end innovation activities on product performance. Journal of Product Innovation Management 30(suppl. 1), 77-92.

McLaughlin P, Bessant J and Smart P (2008) Developing an organisation culture to facilitate radical innovation. International Journal of Technology Management 44, 298. Available at http://www.inderscience.com/link.php? id=21041.

Mulder I and Stappers P (2009) Co-Creating in Practice: Results and Challenges. In 15th International Conference on Concurrent Enterprising, ICE'2010 “Collaborative Innovation: Emerging Technologies, Environments and communities." Leiden.

Nicholas J (2014) An Investigation into the Practices and Underlying Factors during the Fuzzy Front End of Radical Innovation. University of Limerick.

O'Connor GC and Rice M (2001) Opportunity recognition and breakthrough innovation in large established firms. California Management Review 43, 95-116.

Padilha CK and Gomes G (2016) Innovation culture and performance in innovation of products and processes: a study in companies of textile industry. RAI Revista de Administração e Inovação 13, 285-294. Available at http://dx.doi.org/10.1016/j.rai.2016.09.004.

Pallot M and Pawar K (2012) A holistic model of user experience for living lab experiential design. 2012 18th International ICE Conference on Engineering, Technology and Innovation, pp. 1-15. Available at http://www.scopus.com/ inward/record.url?eid=2-s2.0-84867904854\&partnerID=tZOtx3y1.

Pallot M, et al. (2010a) Living Lab research landscape: from user centred design and user experience towards user cocreation. Technology Innovation Management Review 1, 19-25.

Pallot M, Martinez-Carreras MA and Prinz W (2010b) Collaborative distance: a framework for distance factors affecting the performance of distributed collaboration. International Journal of e-Collaboration 6, 1-32.

Pallot M et al. (2012) Innovation gaming: an immersive experience environment enabling co-creation. In Handbook of Research on Serious Games as Educational. Hershey: IGI Global, pp. 1-24.

Pihlajamaa M. (2017) Going the extra mile: Managing individual motivation in radical innovation development. Journal of Engineering and Technology Management - JET-M 43, 48-66.

Rajapathirana RPJ and Hui Y (2017) Relationship between innovation capability, innovation type, and firm performance. Journal of Innovation \& Knowledge, 51, 1-16. 
Ramaswamy V and Gouillart F (2010) Building the Co-Creative Enterprise. Harvard Business Review 88, 100-109,150.

Reid SE and De Brentani U (2004) The fuzzy front end of new product development for discontinuous innovations: a theoretical model. Journal of Product Innovation Management 21, 170-184.

Rihova I, et al. (2013) Social layers of customer-to-customer value co-creation. Journal of Service Management 24, 553-566.

Rolstadås A, Henriksen B and O'Sullivan D (2012) The innovation process. In Manufacturing Outsourcing: A Knowledge Perspective. London: Springer London, pp. 129-144. Available at https://doi.org/10.1007/978-1-44712954-7_15.

Salminen J et al. (2011) Evaluating user involvement within living labs through the use of a domain landscape. 2011 17th International Conference on Concurrent Enterprising.

Sanders EB-N (2008) An evolving map of design practice and design research. Interactions 15, 13-17. Available at http://portal.acm.org/citation.cfm? $\mathrm{id}=1409040.1409043$.

Sanders EB-N and Stappers PJ (2008) Co-creation and the new landscapes of design. CoDesign 4, 5-18. Available at http://www.tandfonline.com/doi/abs/ 10.1080/15710880701875068.

Scapin DL et al. (2012) User experience: Buzzword or new paradigm? ACHI 2012 - 5th International Conference on Advances in ComputerHuman Interactions, (January), pp. 336-341. Available at http://www. scopus.com/inward/record.url?eid=2-s2.0-84883218765\&partnerID=40\&md5= 744ea909df799253f848d5c5953ff24e.

Slater SF, Mohr JJ and Sengupta S (2014) Radical product innovation capability: literature review, synthesis, and illustrative research propositions. Journal of Product Innovation Management 31, 552-566. Available at http://dx.doi.org/10.1111/jpim.12113.

Torgerson WS (1958) Theory and Methods of Scaling. Oxford: Wiley, ed.

Utterback J (1994) Mastering the Dynamics of Innovation: How Companies can Seize Opportunities in the Face of Technological Change. Boston: Harvard Business School Press.

Verworn B, Herstatt C and Nagahira A (2008) The Fuzzy Front End of Japanese New Product Development Projects: Impact on Success and Differences between Incremental and Radical The fuzzy front end of Japanese new product development projects : impact on success and differences between incremental a. (October). pp. 1-19.

Von Hippel E (1986) Lead users: a source of novel product concepts. Management Science 32, 791-805.

Von Hippel E (2005) Democratizing innovation. Cambridge: MIT Press, ed.

Zhang Q and Doll WJ (2001) The fuzzy front end and success of new product development: a causal model. European Journal of Innovation Management 4, 95-112.

Adrien Lecossier, M.Eng, Ph.D., is research associate at "Laval Arts et Metiers Institute" within the "Presence \& Innovation" research team (LAMPA Lab,
EA1427). After had begun his career at the VNIIM Institute in Saint Petersburg, he recently accomplished his work on the radical innovation management proposing a new model which connects human-centred approaches with economical approaches. Considered as the opening of a new territory to explore, this model has made Adrien Lecossier one of the pioneers of radical innovation management in France. Concerned about the industrial applicability of his work, he always keeps a foot in the industrial environment where he officiates as Head of Innovation especially at Esterline or more recently at Primagaz-SHV Energy.

Marc Pallot, M.Eng, Ph.D., is a senior research associate at the Centre for Concurrent Enterprise, University of Nottingham, UK; and at "Laval Arts et Metiers Institute" within the "Presence \& innovation" research team (LAMPA Lab, EA1427), France. He is also adjunct faculty member at the Jyvaskyla University of Applied Sciences, Finland. He teaches "User Driven Innovation" and "Innovation Management". Marc was also a member of the AxIS Team at INRIA Sophia Antipolis where he worked on a landscape study at the crossroads of Future Internet, Living Labs and Smart Cities. He has about 20 years industrial experience within Innovation, $\mathrm{R} \& \mathrm{D}$, and product marketing \& engineering in various sectors such as Telecom, Avionics, Defense, Engineering and CAD/CAM/PDM. He was formerly Vice-President for Product Marketing \& Engineering at Win Technology; a software house specialized in A.I. based Workflow and Product Data Management.

Pascal Crubleau, Senior Lecturer, PhD from the University of Angers is renowned for his research work in the field of Innovation. He is an active member of the LAMPA Lab's "Presence \& Innovation" research team (EA1427). His research and teaching fields are based on technological innovation and the product design process. He has put his expertise at the service of the French Ministry of National Education since 2011 as part of the definition of a technological innovation teaching programme at the secondary level. He is the co-author of a software program for learning innovation principles: "Le guide de l'innovation" ([http://www.ingera.com)] www.ingera.com).

Simon Richir, M.Eng, Ph.D., is one of the pioneers and the most recognized leaders in French VR research and its practical application. Professor at ENSAM (Arts \& Metiers ParisTech), the renowned French School of Engineering, Simon Richir is also the head of "Laval Arts et Metiers Institute" and "Presence \& innovation" research team (LAMPA Lab, EA1427). His research and teaching activities concentrate on technological innovation, engineering design process, ideation, innovative projects, and innovative uses of new advanced technologies such as Virtual Reality or Augmented Reality. In addition to these activities, he is also the co-founder and the present scientific chair of one of the world's most prestigious international events in Virtual Reality: the annual Laval Virtual International Conference. 


\section{Appendix A}

Overall UX-FFE Construct including 4 levels, namely: dimensions, experience, elements and properties.

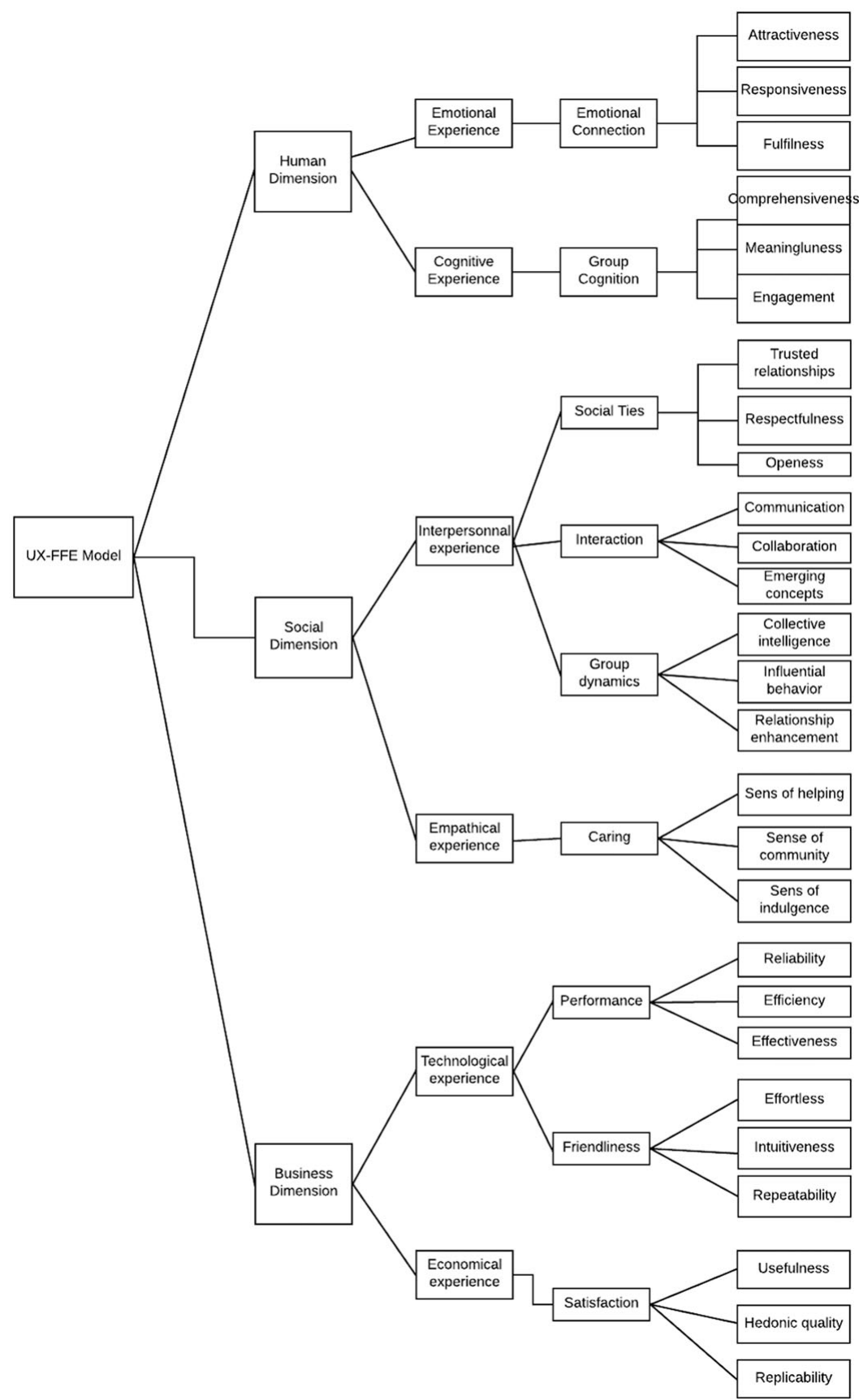

